\title{
NEW RESULTS CONCERNING THE HIERARCHICAL CONTROL OF LINEAR AND SEMILINEAR PARABOLIC EQUATIONS
}

\author{
Bianca M.R. Calsavara ${ }^{1, *}$, Enrique Fernández-Cara ${ }^{2, * *}$, \\ LuZ DE TeresA ${ }^{3,4, * * *, * * * *}$ @ AND José ANTONIO VILLA ${ }^{3, * * * * *}$
}

\begin{abstract}
This paper deals with the application of multiple strategies to control some parabolic PDEs. We assume that we can act on the system through a hierarchy of distributed controls: with a first control (a follower), we drive the state exactly to zero; then, with an additional control (the leader), we minimize a prescribed cost functional. That means that we invert the roles played by leaders and followers in the recent literature. We study linear and semilinear problems. More precisely, we prove the existence (and uniqueness in the linear case) of a leader-follower couple. Then, we deduce an appropriate optimality system that must be satisfied by the controls and the corresponding state and adjoint states. We also indicate some generalizations to other controls, PDEs and systems. In particular, we establish similar existence and optimality results for hierarchical-biobjective (Pareto-Stackelberg) control problems, where there are two cost functionals and two independent leader controls whose main task is to find an associated Pareto equilibrium and one common follower in charge of null controllability.
\end{abstract}

Mathematics Subject Classification. 35Q93, 49J20, 49K20, 93A13, 93B05, 93C20.

Received December 9, 2020. Accepted February 2, 2022.

\section{INTRODUCTION}

The aim of this paper is to get "simultaneously" optimal control and controllability results for linear and semilinear heat equations with multiple (and hierarchical) strategies. We assume that we can act on the system through a hierarchy of distributed controls and, after fixing the cost functional and the desired controllability property, we look for existence, uniqueness and characterization (i.e. optimality) results.

\footnotetext{
* Partially supported by 88887.310561/2018-00 (CAPES, Brazil).

** Partially supported by grant PID2020-114976GB-100 (DGI-MINECO, Spain).

**** Partially supported by DGAPA-UNAM PAPIIT-IN100919.

***** Partially supported by Conacyt, Mexico.

Keywords and phrases: Stackelberg strategies, optimal control, controllability, carleman inequalities, linear and semilinear parabolic PDEs.

1 IMECC, State University of Campinas, 13083-859, Campinas, SP, Brazil.

2 Dpto. EDAN and IMUS, University of Sevilla, Aptdo. 1160, 41080 Sevilla, Spain.

3 Instituto de Matemáticas, Universidad Nacional Autónoma de México.

4 Dpto. de Matemática, Universidade Federal da Paraíba, Brazil.

*** Corresponding author: deteresal@gmail.com
} 
In classical control theory, we usually deal with a state equation or system and just one control and we try to find control-state couples that achieve a predetermined goal. Sometimes, the goal is to minimize a cost functional in a prescribed family of admissible controls and/or states; this is the standard optimal control viewpoint. In other cases, the goal is to reach a desired state at the final time of evolution; this happens when we investigate the controllability properties of the system.

An interesting situation arises when several (maybe conflictive or contradictory) objectives are considered. This may happen, for example, if the cost function is the sum of several terms and it is not completely clear which is the most suitable average. It can also be expectable to have more than one control acting on the equation. In these cases, we are led to consider multi-objective and/or hierarchic control problems and the true goal becomes to find one or several equilibria.

Recently, there has been some work on the subject. Thus, in the context of the control of time-dependent PDEs, in the seminal papers by Lions [15, 16], Díaz and Lions [9] and Díaz [8], the authors considered the linear heat equation; they introduced Stackelberg-Pareto and Stackelberg-Nash strategies, leading to a hierarchy of controls with states approximately driven to a given target. In [19, 20], Ramos, Glowinski and Periaux studied Nash equilibria from both the theoretical and numerical viewpoints for linear parabolic PDEs and the Burgers equation. Later, similar Stackelberg-Nash strategies were applied to the Stokes systems by Guillén-González and others in [13]. Let us also mention the works [3-5], where hierarchic exact controllability results were established for linear and semilinear heat equaions.

In all these papers, the main idea is to work with one main control (the leader) and one or several secondary controls (the followers). For each possible leader, the associated followers try to minimize a functional (or reach an equilibrium if there are several cost objective functions).

Then, the leader is chosen such that the corresponding state takes a prescribed value (exactly or approximately) at final time (a controllability constraint). This kind of problems can be illustrated by relevant social situations, where the leader represents (for instance) the State Government and is the major responsible to attain a concrete state favorable to social purposes, while the followers are more interested in finding optimal options compatible with the laws.

This paper deals with a similar (but different) strategy, trying again to accomplish optimal control and controllability tasks with a hierarchy of controls. This time, however, the controllability goal will be commended to the follower, while the choice of the leader will be defined by the solution to an optimal control problem.

A motivation can be found, for example, in environmental sciences, when a Government wants to maintain pollution at a minimal level and the followers want to achieve a particular goal.

It will be seen that this makes the problem more difficult to handle (essentially because we must work all the time in a very restrictive class of leader controls).

\subsection{The problems and their motivations}

Let $\Omega \subset \mathbf{R}^{N}$ be a bounded connected open set with regular boundary. Let $T>0$ be given and let us consider the cylinder $Q:=\Omega \times(0, T)$, with lateral boundary $\Sigma:=\partial \Omega \times(0, T)$. In the sequel, we will denote by $C$ a generic positive constant. Sometimes, we will indicate the data on which it depends by writing $C(\Omega), C(\Omega, T)$, etc. The usual norm and scalar product in $L^{2}(\Omega)$ will be respectively denoted by $\|\cdot\|$ and $(\cdot, \cdot)$.

Our main interest is, in a few words, to solve some optimal control problems where, additionally, the state is driven to rest. For simplicity, we will assume for the moment that only two controls are applied (one leader and one follower) but, as shown below, similar considerations hold for systems with a higher number of controls.

We will consider systems of the form

$$
\begin{cases}y_{t}-\Delta y+a(x, t) y=f 1_{\mathcal{O}}+v 1_{\omega} & \text { in } Q \\ y=0 & \text { on } \Sigma, \\ y(\cdot, 0)=y_{0} & \text { in } \Omega\end{cases}
$$


and

$$
\begin{cases}y_{t}-\Delta y+F(y)=f 1_{\mathcal{O}}+v 1_{\omega} & \text { in } Q \\ y=0 & \text { on } \Sigma, \\ y(\cdot, 0)=y_{0} & \text { in } \Omega,\end{cases}
$$

where $f$ and $v$ are the controls, $y$ is the state, $a \in L^{\infty}(Q), F$ is a $C^{1}$ globally Lipschitz-continuous function with $F(0)=0$ and $y_{0} \in L^{2}(\Omega)$ is prescribed. In (1.1) and (1.2), the set $\omega \subset \Omega$ is the main control domain and $\mathcal{O} \subset \Omega$ is the secondary control domain (both are supposed to be small); in order to avoid ambiguity, we will assume that $\mathcal{O}$ and $\omega$ are disjoint; $1_{\mathcal{O}}$ and $1_{\omega}$ are the characteristic functions of $\mathcal{O}$ and $\omega$, respectively; $f$ is the follower and $v$ is the leader.

Let us describe the considered hierarchic problem in the case of (1.1).

Let $\omega_{d} \subset \Omega$ be a non-empty open set, representing an observation domain for the leader. We will consider the secondary functional

$$
S(v ; f):=\frac{1}{2} \iint_{Q} \rho^{2}|y|^{2}+\frac{1}{2} \iint_{\mathcal{O} \times(0, T)} \rho_{0}^{2}|f|^{2},
$$

where $\rho$ and $\rho_{0}$ are appropriate weights that blow up as $t \rightarrow T^{-}$, that is, as $t$ grows to $T$, see (1.10)-(1.12) for the precise definitions. Note that, if $S(v ; f)<+\infty$, we necessarily have $y(x, T) \equiv 0$. We will also consider the main functional

$$
P(v ; f):=\frac{\alpha}{2} \iint_{\omega_{d} \times(0, T)}\left|y-y_{d}\right|^{2}+\frac{\mu}{2} \iint_{\omega \times(0, T)} \rho_{0}^{2}|v|^{2},
$$

where $\alpha$ and $\mu$ are positive constants with $\alpha+\mu=1$ and $y_{d}=y_{d}(x, t)$ is a given function (a desired observation).

The following spaces will be used:

$$
\begin{gathered}
\mathcal{U}:=\left\{v: \rho_{0} v \in L^{2}(\omega \times(0, T))\right\} \\
\mathcal{Y}:=\left\{y: \rho y \in L^{2}(Q)\right\}, \quad \mathcal{F}:=\left\{f: \rho_{0} f \in L^{2}(\mathcal{O} \times(0, T))\right\} .
\end{gathered}
$$

The natural norms in $\mathcal{U}$ and $\mathcal{F}$ will be respectively denoted by $\|\cdot\|_{\mathcal{U}}$ and $\|\cdot\|_{\mathcal{F}}$.

The control process can be described as follows:

1. We associate to each leader $v \in \mathcal{U}$ the unique solution $f[v]$ to the following extremal problem:

$$
\left\{\begin{array}{l}
\text { Minimize } S(v ; f) \\
\text { Subject to } f \in \mathcal{F} .
\end{array}\right.
$$

In view of the behavior of $\rho$ near $t=T$, the state $y$ associated to $v$ and $f[v]$ must necessarily satisfy the null controllability property

$$
y(\cdot, T)=0 \quad \text { in } \quad \Omega .
$$

2. Then, we look for admissible controls $\hat{v} \in \mathcal{U}$ satisfying

$$
P(\hat{v} ; f[\hat{v}])=\min _{v} P(v ; f[v])
$$


There are several reasons to introduce $\rho$ and $\rho_{0}$. First, note that they ensure the null controllability constraint $y(x, T) \equiv 0$ automatically. Secondly, they prevent $v$ to present undesirable oscillations as $t \rightarrow T^{-}$; indeed, it is well known that this can happen if, for instance, we search for minimal $L^{2}$-norm null controls. A third reason is that, as indicated in Proposition 2.3, a good choice of these weights reduces the search of the follower $f[v]$ and the associated state $y$ to the solution of a well posed Lax-Milgram problem; see (2.3) and (2.4).

There are other ways to find a follower corresponding to a prescribed leader $v$; see [6] to this regard. However, to our knowledge, in order to ensure a good behavior of the mapping $v \mapsto f[v]$, the best strategy is to solve secondary extremal problems of the kind (1.6).

Observe that, if the function $v \mapsto P(v ; f[v])$ is differentiable in the space $\mathcal{U}$ of admissible leader controls, then (1.8) implies

$$
\left.\frac{\mathrm{d}}{d v} P(v ; f[v])\right|_{v=\hat{v}}=0
$$

This property will be crucial for the characterization of the optimal control $\hat{v}$ and the associated $f[\hat{v}]$.

Note also that, after a very simple change of variable, we can also consider a hierarchic problem in which, instead of (1.7), we require

$$
y(\cdot, T)=\bar{y}(\cdot, T) \text { in } \Omega,
$$

where $\bar{y}$ is an uncontrolled solution to (1.1).

Consequently, it is also meaningful to look for optimal leaders and associated followers that drive the solution to (1.1) exactly to a prescribed trajectory $t \rightarrow \bar{y}(\cdot, t)$ at time $T$.

In the case of the semilinear system (1.2), we can consider hierarchic control problems of the same kind. However, their formulation is more complicated and will be delayed to the following section. Indeed, in that case, (1.6) possesses in general not one but probably several solutions and (1.8) needs a reformulation.

Several motivations can be found for these control problems:

- If $y=y(x, t)$ is viewed as a temperature distribution in a body, we can interpret that our intention is to drive $y$ to a desired $\bar{y}$ at time $T$ by heating and cooling (acting only on the small subdomains $\mathcal{O}$ and $\omega$ ), trying at the same time to keep reasonable temperatures in $\omega_{d}$ during the whole time interval $(0, T)$. For instance, let us take $a \equiv 0$ and $\bar{y}(x, t) \equiv y_{T}$ (a nonnegative constant). Then, we can think of a two-piece apartment with an air conditioner in the bedroom and another one in the living room, respectively playing the roles of the follower and leader controls. The aims are to get a temperature close to a desired target in the living room (for example) along $(0, T)$ and ensure that, at the end of the day (at $t=T$ ), one has $y(x, T) \equiv y_{T}$ everywhere.

- The same control strategy makes sense in the context of fluid mechanics. Thus, we can replace (1.1) by similar Stokes or Navier-Stokes systems and take into consideration similar hierarchic problems. We can interpret that we act on the system through mechanical forces applied on $\mathcal{O}$ and $\omega$ and the goal is to reach $\bar{y}$ at time $T$ keeping the velocity field not too far from $y_{d}$ in $\omega_{d} \times(0, T)$.

- In the framework of mathematical finance, this can also be of interest. For instance, it is well known that the price of an European call option is governed by a backwards in time PDE close to (1.2). Now, the independent variable $x$ must be interpreted as a vector indicating the prices of the stocks and $t$ is in fact the reverse of time (we fix a situation at $t=T$ and we want to know what to do in order to arrive at this situation from a well chosen state). In this regard, it is natural and can be interesting to control the solution to the system with the composed action of several agents, each of them corresponding to a different range of values of $x$. For example, with $N=1$ (only one stock) and $\Omega=(0, L)$, we can take $\omega_{d}=\Omega, \mathcal{O}=\left(0, L_{0}\right)$ and $\omega=\left(L_{1}, L\right)$ for some $L_{0}$ and $L_{1}$ with $0<L_{0} \leq L_{1}<L$. This means that the "important" decisions are taken when the stock price is large and this determines what to do when the 
price is low. This way, we intend to get an option price close to a desired target along $(0, T)$, subject to the controllability requirement at $t=0$.

For further information on the modeling and control of these phenomena, see for instance [7, 21, 22].

\subsection{Main results}

Before stating our main results, let us specify once for all the weight functions $\rho$ and $\rho_{0}$. We will see later that their definitions are motivated by well known controllability results for (1.1) in suitable spaces.

Let $\eta_{0}=\eta_{0}(x)$ be a function satisfying

$$
\left\{\begin{array}{l}
\eta_{0} \in C^{2}(\bar{\Omega}), \eta_{0}>0 \text { in } \Omega, \eta_{0}=0 \text { on } \partial \Omega \\
\left|\nabla \eta_{0}\right|>0 \text { in } \bar{\Omega} \backslash \omega
\end{array}\right.
$$

With our assumptions on $\Omega$, such a function $\eta_{0}$ always exists (see Lem. 1.1, p. 4 in [11]). Then, let us introduce the weight functions

$$
\begin{gathered}
\sigma(x, t):=\frac{e^{4 \lambda\left\|\eta^{0}\right\|_{\infty}}-e^{\lambda\left(2\left\|\eta^{0}\right\|_{\infty}+\eta^{0}(x)\right)}}{\ell(t)}, \quad \xi(x, t):=\frac{e^{\lambda\left(2\left\|\eta^{0}\right\|_{\infty}+\eta^{0}(x)\right)}}{\ell(t)}, \\
\rho:=e^{s \sigma}, \quad \rho_{0}:=(s \xi)^{-3 / 2} \lambda^{-2} \rho, \quad \rho_{1}:=(s \xi)^{-1 / 2} \lambda^{-1} \rho, \quad \rho_{2}:=(s \xi)^{1 / 2} \rho,
\end{gathered}
$$

where $\ell \in C^{\infty}([0, T])$ satisfies

$$
T^{2} / 8 \leq \ell(t) \geq T^{4} / 4 \text { in }[0, T / 2] \text { and } \ell(t)=t(T-t) \text { in }[T / 2, T]
$$

and $\lambda, s>0$ are large enough. In fact, the required values of $\lambda$ and $s$ will be fixed in accordance with Theorem 2.1 below in the linear case and satisfying (3.1) for semilinear systems.

At first sight, the choice of $\rho$ and $\rho_{0}$ may seem too artificial. However, we do need weights like these, since they appear in the Carleman inequality (2.1) used in the proofs of the main results.

In the case of (1.1), the following holds:

Theorem 1.1. Let us consider the linear system (1.1), where $a \in L^{\infty}(Q)$ and $y_{0} \in L^{2}(\Omega)$.

1. For every $v \in \mathcal{U}$, there exists exactly one solution $f[v]$ to (1.6).

2. Let us set $J(v):=P(v ; f[v])$. Then there exists exactly one minimizer $\hat{v}$ of $J$ in $\mathcal{U}$ and, consequently, one associated follower $f[\hat{v}]$ such that (1.7) holds.

We will see in Section 2 that the minimizer $\hat{v}$ satisfies, together with the corresponding $f[\hat{v}]$, the associated state $\hat{y}$ and some additional (adjoint) variables, an appropriate optimality system; the details are given in Theorem 2.6. On the other hand, in Section 5.4, we will see that results similar to Theorem 1.1 (and Thm. 2.6) can also be obtained for many other linear systems, including the well known Stokes equations; see Theorem 5.3.

In the semilinear case, with $F$ being a Lipschitz-continuous function, we can consider similar controllability questions. However, it is important to note that, now, we lose the convexity of the functionals $S$ and $P$ and this introduces several nontrivial difficulties.

Thus, for each $v \in \mathcal{U}$, we can consider the extremal problem (1.6), where $S$ is again given by (1.3) but, now, $y$ is the unique solution to (1.2). We will denote by $\Phi[v]$ the family of solutions to (1.6). In this case, we will look for a leader $\hat{v}$ and an associated follower $\hat{f}$ such that, instead of (1.8), one has:

$$
P(\hat{v} ; \hat{f})=\min _{v, f} P(v ; f),
$$

where we minimize in the set of pairs $(v, f)$ with $v \in \mathcal{U}$ and $f \in \Phi[v]$.

The following holds: 
Theorem 1.2. Let us consider the semilinear system (1.2), where $F: \mathbf{R} \mapsto \mathbf{R}$ is $C^{1}$ and globally Lipschitzcontinuous and $F(0)=0$ and $y_{0} \in L^{2}(\Omega)$.

1. For every $v \in \mathcal{U}$, the set $\Phi[v]$ is non-empty, that is, there exists at least one solution to (1.6).

2. On the other hand, the extremal problem (1.13), where the minimum is extended to all couples $(v, f)$ with $v \in \mathcal{U}$ and $f \in \Phi[v]$, possesses at least one solution $(\hat{v}, \hat{f})$.

In this paper, we also analyze if a result like Theorem 1.1 holds true when the leader is constrained to belong to an appropriate convex set $\mathcal{U}_{\text {ad }} \subset L^{2}(\omega \times(0, T))$. Thus, let $I$ be a non-empty closed interval with $0 \in I$, let us take

$$
\mathcal{U}_{\text {ad }}=\{v \in \mathcal{U}: v(x, t) \in I \text { a.e. }\}
$$

and let us suppose that the minimization in (1.8) is subject to the restriction $v \in \mathcal{U}_{\mathrm{ad}}$. The control result is then the following:

Theorem 1.3. Let us consider the linear system (1.1), where $a \in L^{\infty}(Q)$ and $y_{0} \in L^{2}(\Omega)$. There exists exactly one minimizer $\hat{v}$ of $J$ in $\mathcal{U}_{\text {ad }}$ and an associated follower $f[\hat{v}]$ such that the corresponding state satisfies (1.7).

In Section 4, we will consider a situation where adequate constraints are imposed not only to the leaders but also to the associated followers; see Remark 4.2.

\subsection{Plan of the paper}

As mentioned above, the main novelty of this paper is that the choice of the follower (resp. the leader) is determined by a controllability (resp. an optimal control) requirement. The analysis and results also hold, after appropriate modifications, when several main cost functionals (and several leader controls) appear and, instead of an extremal problem, we look for related equilibria. All this will be explained below.

The rest of the paper is organized as follows.

In Section 2, we prove Theorem 1.1, which concerns the linear case. This result will be strongly used in the remaining sections. We will also establish a characterization result for the optimal leader-follower-state triplet (see Thm. 2.6). In Section 3, we prove Theorem 1.2; we also deduce an optimality system that must be satisfied by any solution to (1.13). Section 4 is devoted to prove Theorem 1.3 and make some additional considerations on hierarchical control with constraints. Finally, we present some additional comments and questions in Section 5.

\section{THE LINEAR CASE}

In this section we prove Theorem 1.1. Thus, let us consider the linear system (1.1), let us introduce the notation

$$
L_{a} y:=y_{t}-\Delta y+a y, \quad L_{a}^{*} p:=-p_{t}-\Delta p+a p
$$

and let the space $\mathcal{P}_{0}$ be given by

$$
\mathcal{P}_{0}:=\left\{p \in C^{2}(\bar{Q}): p=0 \text { on } \Sigma\right\} .
$$

We will need the following symmetric bilinear forms on $\mathcal{P}_{0}$ associated to the coefficients $a \in L^{\infty}(Q)$ :

$$
m(a ; p, q):=\iint_{Q}\left(\rho^{-2} L_{a}^{*} p L_{a}^{*} q+1_{\mathcal{O}} \rho_{0}^{-2} p q\right) .
$$

From the unique continuation property satisfied by the solutions to homogeneous heat equations, we know that all these bilinear forms are in fact a scalar products (actually, it will be seen below that they are equivalent). 
In the sequel, we will denote by $\mathcal{P}$ the completion of $\mathcal{P}_{0}$ associated to $m(0 ; \cdot, \cdot)$. It is known that the functions in $\mathcal{P}$, their first and second spatial derivatives and their first time derivatives are locally square integrable in $\Omega \times(0, T-\delta)$ for all small $\delta>0$. More precisely, we have the following Carleman inequality:

Theorem 2.1. There exist positive constants $\lambda_{0}, s_{0}$ and $C_{0}$, only depending on $\Omega, \mathcal{O}$ and $T$, such that, if we take $\lambda=\lambda_{0}$ and $s=s_{0}$ in (1.11), any $p \in \mathcal{P}$ satisfies

$$
\iint_{Q}\left[\rho_{2}^{-2}\left(\left|p_{t}\right|^{2}+|\Delta p|^{2}\right)+\rho_{1}^{-2}|\nabla p|^{2}+\rho_{0}^{-2}|p|^{2}\right] \leq C_{0} m(0 ; p, p) .
$$

Furthermore, $\lambda_{0}$ and $s_{0}$ can be found arbitrarily large.

The proof of this result is given in [11]; see also [10] for more details on the constants.

A consequence of (2.1) is that the (adjoint of the) heat equation in (1.1) is observable. In other words, there exists a constant $C$ only depending on $\Omega, \mathcal{O}, T$ and $a$ such that, if $\varphi \in L^{2}\left(0, T ; H_{0}^{1}(\Omega)\right) \cap C^{0}\left([0, T] ; L^{2}(\Omega)\right)$ and $L_{a}^{*} \varphi=0$ in $Q$, then

$$
\|\varphi(\cdot, 0)\|^{2} \leq C \iint_{\mathcal{O} \times(0, T)}|\varphi|^{2}
$$

These estimates ensure the null controllability property of the heat equation with controls in $L^{2}(\mathcal{O} \times(0, T))$; see for instance [10].

In the remainder of this section, it will be assumed that $\lambda=\lambda_{0}$ and $s=s_{0}$. A straightforward consequence of Theorem 2.1 is the following:

Corollary 2.2. There exist positive constants $K_{0}$ and $K_{1}$, only depending on $\Omega, \mathcal{O}, T$ and $\|a\|_{L^{\infty}(Q)}$, such that the following holds:

$$
K_{0} m(0 ; p, p) \leq m(a ; p, p) \leq K_{1} m(0 ; p, p) \quad \forall p \in \mathcal{P} .
$$

In the following result, we recall that, for any admissible $v$, the associated follower is well defined:

Proposition 2.3. Let $v$ be given in $\mathcal{U}$. Then, there exists exactly one solution $f[v] \in \mathcal{F}$ to the null controllability problem (1.6), where $S$ is given by (1.3) and $y$ is the solution to (1.1). Furthermore, one has

$$
f[v]=-\left.\rho_{0}^{-2} p\right|_{\mathcal{O} \times(0, T)}, \quad y=\rho^{-2} L_{a}^{*} p,
$$

where $p$ is the unique solution to the linear problem

$$
\left\{\begin{array}{l}
m(a ; p, q)=\ell(v ; q) \\
\forall q \in \mathcal{P}, \quad p \in \mathcal{P}
\end{array}\right.
$$

and we have used the notation

$$
\ell(v ; q):=\iint_{\omega \times(0, T)} v q+\int_{\Omega} y_{0}(x) q(x, 0) \mathrm{d} x .
$$

Proof. We use here the nowadays well known Fursilkov-Imanuvilov approach to null controllability, see [11].

First, remark that the Lax-Milgram's Theorem can be applied to $(2.4)$. Indeed, $m(a ; \cdot, \cdot)$ is obviously continuous and coercive in $\mathcal{P}$. On the other hand, in view of (2.1), the functions $p \in \mathcal{P}$ satisfy

$$
p 1_{[0, T / 2]} \in L^{2}\left(0, T / 2 ; H^{2}(\Omega)\right), \quad p_{t} 1_{[0, T / 2]} \in L^{2}\left(0, T / 2 ; L^{2}(\Omega)\right)
$$


and, consequently,

$$
p 1_{[0, T / 2]} \in C^{0}\left([0, T / 2] ; H_{0}^{1}(\Omega)\right)
$$

with appropriate continuous embeddings. Therefore, the linear mapping $p \mapsto p(\cdot, 0)$ is well defined and continuous from $\mathcal{P}$ into $H_{0}^{1}(\Omega)$; this shows that the right hand side in (2.4) is a bounded linear form on $\mathcal{P}$.

Let $f[v]$ and $y$ be given by (2.3). Then, it is not difficult to check that $f[v] \in \mathcal{F}, y$ is the state associated to $v$ and belongs to $\mathcal{Y}$ and $f[v]$ solves (1.6). The function $f \mapsto S(v ; f)$ is coercive, lower semi-continuous, convex and proper in $\mathcal{F}$. It is also quadratic and strictly convex in its domain. Consequently, we also have that $f[v]$ is the unique solution to (1.6).

This ends the proof.

Observation 2.4. The previous argument yields the estimates

$$
|\ell(v ; p)| \leq C\left(\|v\|_{\mathcal{U}}+\left\|y_{0}\right\|\right) m(0 ; p, p)^{1 / 2} \quad \forall p \in \mathcal{P}
$$

for some $C$ only depending on $\Omega, \mathcal{O}, T$ and $\|a\|_{L^{\infty}(Q)}$. This leads to the following estimates of $p, f[v]$ and the associated state $y$ :

$$
m(0 ; p, p)^{1 / 2}+\|f[v]\|_{\mathcal{F}}+\|y\|_{\mathcal{Y}} \leq C\left(\|v\|_{\mathcal{U}}+\left\|y_{0}\right\|\right) .
$$

Actually, (2.4) may be viewed as a boundary problem for a PDE that is fourth-order in space and second-order in time. Specifically, $p$ solves (2.4) if and only if $p \in \mathcal{P}$ and one has

$$
\begin{cases}L_{a}\left(\rho^{-2} L_{a}^{*} p\right)+\rho_{0}^{-2} p 1_{\mathcal{O} \times(0, T)}=v 1_{\omega \times(0, T)} & \text { in } Q, \\ p=0, \quad \rho^{-2} L_{a}^{*} p=0 & \text { on } \Sigma, \\ \left.\rho^{-2} L_{a}^{*} p\right|_{t=0}=y_{0},\left.\quad \rho^{-2} L_{a}^{*} p\right|_{t=T}=0 & \text { in } \Omega .\end{cases}
$$

Clearly, in order to prove the existence of a solution to (1.8), it is convenient to analyze the behavior of the function $v \mapsto P(v ; f[v])$ and, more precisely, its convexity and differentiability properties. This is the objective of the following result, whose proof is elementary:

Proposition 2.5. The real-valued function $v \mapsto P(v ; f[v])$ is well defined, $C^{1}$, strictly convex and coercive on $\mathcal{U}$.

The proof of Theorem 1.1 is a direct consequence of Propositions 2.3 and 2.5. Indeed, the properties of the mapping $v \mapsto f[v]$ and the functional in (1.4) guarantee that there exists exactly one control $v \in \mathcal{U}$ satisfying (1.8).

To end this section, let us establish a characterization result:

Theorem 2.6. The unique solution $\hat{v}$ to (1.8) satisfies, together with the associated $\hat{y}, \hat{p}, \hat{\phi}$ and $\hat{\psi}$, the following optimality system:

$$
\begin{gathered}
\begin{cases}\hat{y}_{t}-\Delta \hat{y}+a(x, t) \hat{y}=f[\hat{v}] 1_{\mathcal{O}}+\hat{v} 1_{\omega} & \text { in } \quad Q, \\
\hat{y}=0 & \text { on } \Sigma, \\
\hat{y}(\cdot, 0)=y_{0} & \text { in } \Omega,\end{cases} \\
\quad f[\hat{v}]=-\left.\rho_{0}^{-2} \hat{p}\right|_{\mathcal{O} \times(0, T)}, \quad \hat{y}=\rho^{-2} L_{a}^{*} \hat{p},
\end{gathered}
$$




$$
\begin{gathered}
\left\{\begin{array}{l}
m(a ; \hat{p}, q)=\iint_{\omega \times(0, T)} \hat{v} q+\int_{\Omega} y_{0}(x) q(x, 0) \mathrm{d} x \\
\forall q \in \mathcal{P}, \quad \hat{p} \in \mathcal{P},
\end{array}\right. \\
\left\{\begin{array}{l}
-\hat{\phi}_{t}-\Delta \hat{\phi}+a(x, t) \hat{\phi}=\alpha\left(\hat{y}-y_{d}\right) 1_{\omega_{d}} \text { in } \quad Q, \\
\hat{\phi}=0 \\
\hat{\phi}(\cdot, T)=0
\end{array} \begin{array}{l}
\text { on } \quad \Omega, \\
m(a ; q, \hat{\psi})=-\iint_{\mathcal{O} \times(0, T)} \rho_{0}^{-2} \hat{\phi} q \\
\forall q \in \mathcal{P}, \quad \hat{\psi} \in \mathcal{P},
\end{array}\right. \\
\hat{v}=-\left.\frac{1}{\mu} \rho_{0}^{-2}(\hat{\psi}+\hat{\phi})\right|_{\omega \times(0, T)} .
\end{gathered}
$$

Proof. Let $v, w \in \mathcal{U}$ and $\varepsilon>0$ be given, let us set set $g:=\frac{1}{\varepsilon}(f[v+\varepsilon w]-f[v])$ and let us introduce the solutions $z, \phi, k$ and $\eta$ to the following problems:

$$
\begin{gathered}
\begin{cases}z_{t}-\Delta z+a(x, t) z=g 1_{\mathcal{O}}+w 1_{\omega} & \text { in } Q, \\
z=0 & \text { on } \Sigma, \\
z(\cdot, 0)=0 & \text { in } \Omega,\end{cases} \\
\begin{cases}-\phi_{t}-\Delta \phi+a(x, t) \phi=\alpha\left(y-y_{d}\right) 1_{\omega_{d}} & \text { in } Q, \\
\phi=0 & \text { on } \Sigma, \\
\phi(\cdot, T)=0 & \text { in } \Omega,\end{cases} \\
\left\{\begin{array}{l}
m(a ; k, q)=\iint_{\omega \times(0, T)} w q \\
\forall q \in \mathcal{P}, \quad k \in \mathcal{P}
\end{array}\right.
\end{gathered}
$$

and

$$
\left\{\begin{array}{l}
m(a ; q, \psi)=-\iint_{\mathcal{O} \times(0, T)} \rho_{0}^{-2} \phi q \\
\forall q \in \mathcal{P}, \quad \psi \in \mathcal{P} .
\end{array}\right.
$$

Then, after some computations, one has

$$
\begin{aligned}
\left.\frac{\mathrm{d}}{\mathrm{d} \varepsilon} P(f[v+\varepsilon w] ; v+\varepsilon w)\right|_{\varepsilon=0} & =\alpha \iint_{\omega_{d} \times(0, T)}\left(y-y_{d}\right) z+\mu \iint_{\omega \times(0, T)} \rho_{0}^{2} v w \\
& =\iint_{\mathcal{O} \times(0, T)} \phi g+\iint_{\omega \times(0, T)}\left(\phi+\mu \rho_{0}^{2} v\right) w
\end{aligned}
$$


Note that $g=-\left.\rho_{0}^{-2} k\right|_{\omega \times(0, T)}$, whence

$$
\iint_{\mathcal{O} \times(0, T)} \phi g=-\iint_{\mathcal{O} \times(0, T)} \rho_{0}^{-2} \phi k=m(a ; k, \psi)=\iint_{\omega \times(0, T)} \psi w .
$$

Consequently, the following identity holds for all $v, w \in \mathcal{U}$ :

$$
\left.\frac{\mathrm{d}}{\mathrm{d} \varepsilon} P(f[v+\varepsilon w] ; v+\varepsilon w)\right|_{\varepsilon=0}=\iint_{\omega \times(0, T)}\left(\phi+\psi+\mu \rho_{0}^{2} v\right) w .
$$

In particular, with $v=\hat{v}$, denoting by $\hat{y}, \hat{\phi}$ and $\hat{\psi}$ the associated state and adjoint states and taking $w$ arbitrary in $\mathcal{U}$, we see that $\hat{\phi}+\hat{\psi}+\mu \rho_{0}^{2} \hat{v}=0$ a.e. in $\omega \times(0, T)$, whence the assertion follows.

\section{THE SEMILINEAR CASE}

This section is mainly devoted to prove Theorem 1.2. We will use arguments similar to those above that lead to existence results for (1.6) and (1.13).

We will also find a necessary condition for optimality, similar to (2.7)-(2.12), that has to be satisfied by any solution to the control problem.

Let us introduce $R:=\sup _{\mathbf{R}}\left|F^{\prime}(r)\right|$. In the sequel, it will be assumed that the weights $\rho$ and $\rho_{0}$ are given by (1.11) with $\lambda=\lambda_{0}$ and $s=s_{0}$, where $\lambda_{0}$ and $s_{0}$ are furnished by Theorem 2.1 and satisfy

$$
s_{0}^{3 / 2}>\sqrt{2} R \lambda_{0}^{-2} \sup _{Q} \xi^{-3 / 2}=\frac{\sqrt{2}}{8} \frac{R T^{3}}{\lambda_{0}^{2} e^{3 \lambda_{0}\left\|\eta^{0}\right\|_{L^{\infty}}}} .
$$

Obviously, there exist constants $K_{1}$ and $K_{2}$, only depending on $\Omega, \mathcal{O}, T$ and $R$, such that $(2.2)$ holds for all $a \in L^{\infty}(Q)$ with $\|a\|_{L^{\infty}(Q)} \leq R$.

\subsection{Proof of Theorem 1.2}

Let us first prove that any admissible leader $v$ possesses at least one follower in $\mathcal{F}$ :

Proposition 3.1. Let $v$ be given in $\mathcal{U}$. Then, there exists at least one solution in $\mathcal{F}$ to the extremal problem (1.6), where $S$ is given by (1.3) and $y$ is the solution to (1.2). Furthermore, any solution $f$ to (1.6) satisfies, together with the associated state $y$ and an additional variable $p \in \mathcal{P}$, the semilinear system (1.2), the identities

$$
f=-\left.\rho_{0}^{-2} p\right|_{\mathcal{O} \times(0, T)} \text { and } y=\rho^{-2} L_{F^{\prime}(y)}^{*} p
$$

and the estimates

$$
m(0 ; p, p)^{1 / 2} \leq C\left(\|v\|_{\mathcal{U}}+\left\|y_{0}\right\|\right) \quad \text { and } \quad\|f\|_{\mathcal{F}}+\|y\|_{\mathcal{Y}} \leq C\left(\|v\|_{\mathcal{U}}+\left\|y_{0}\right\|\right)
$$

for some $C$ only depending on $\Omega, \mathcal{O}, T$ and $R$.

Proof. Let us first see that there exist controls $f \in \mathcal{F}$ such that $S(v ; f)<+\infty$. Indeed, let us denote by $F_{0}$ the function given by

$$
F_{0}(\xi)=\frac{F(\xi)}{\xi} \text { if } \xi \neq 0, \quad F_{0}(0)=F^{\prime}(0)
$$

Obviously, $F_{0}(\xi)$ is uniformly bounded in $\mathbf{R}$. 
For each $z \in L^{2}(Q)$, we will denote by $\Lambda(z)$ the unique solution $y_{z}$ to the linear problem

$$
\begin{cases}y_{t}-\Delta y+F_{0}(z) y=f 1_{\mathcal{O}}+v 1_{\omega} & \text { in } Q, \\ y=0 & \text { on } \Sigma, \\ y(\cdot, 0)=y_{0} & \text { in } \Omega,\end{cases}
$$

where $f$ is the unique solution to (1.6) with $a=F_{0}(z)$. Let us denote this control by $f_{z}$.

The existence and uniqueness of $f_{z}$ is a consequence of the arguments in Section 2 (see Prop. 2.3). Furthermore, since the $F_{0}(z)$ are uniformly bounded in $L^{\infty}(Q)$, the controls $f_{z}$ are uniformly bounded in $\mathcal{F}$ and the associated states $y_{z}$ belong (among other things) to a fixed compact set in $L^{2}(Q)$.

Thus, the mapping $z \mapsto \Lambda(z)$ is well-defined, continuous and compact in $L^{2}(Q)$ and maps the whole space into a ball. In view of Schauder's Theorem, $\Lambda$ possesses at least one fixed-point $\tilde{y}$. If we set $\tilde{f}:=f_{\tilde{y}}$, then we obviously have $S(v ; \tilde{f})<+\infty$.

Now, let $\left\{f^{n}\right\}$ be a minimizing sequence for (1.6). It is clear that the $f^{n}$ (resp. $y^{n}$ ) are uniformly bounded in $\mathcal{F}$ (resp. $\mathcal{Y}$ ). Consequently, it can be assumed that they converge weakly in $\mathcal{F}$ to some $f$ and the corresponding states $y^{n}$ converge strongly in $L^{2}(Q)$ to the associated $y$. From the weak lower semicontinuity of the functionals

$$
y \mapsto \iint_{Q} \rho^{2}|y|^{2} \text { and } f \mapsto \iint_{\mathcal{O} \times(0, T)} \rho_{0}^{2}|f|^{2},
$$

we easily deduce that $f$ minimizes (1.6). Hence, there exists at least one solution to this extremal problem.

Let us prove that any solution to (1.6) satisfies (3.2) for some $p \in \mathcal{P}$.

Thus, let $f \in \mathcal{F}$ be a solution to (1.6) and let us denote by $y$ the corresponding solution to (1.2). Let us introduce the solution $\bar{y}$ to the auxiliary problem

$$
\begin{cases}\bar{y}_{t}-\Delta \bar{y}=0 & \text { in } \quad Q \\ \bar{y}=0 & \text { on } \quad \Sigma \\ \bar{y}(\cdot, 0)=y_{0} & \text { in } \quad \Omega\end{cases}
$$

the linear mapping $H_{0}: L^{2}(Q) \mapsto L^{2}(Q)$ with $w=H_{0} k$ if and only if

$$
\begin{cases}w_{t}-\Delta w=k & \text { in } \quad Q \\ w=0 & \text { on } \quad \Sigma, \\ w(\cdot, 0)=0 & \text { in } \Omega\end{cases}
$$

and the nonlinear mapping $M: \mathcal{Y} \times \mathcal{F} \mapsto L^{2}(Q)$, with

$$
M(y, f):=y-H_{0}\left(v 1_{\omega \times(0, T)}+f 1_{\mathcal{O} \times(0, T)}-F(y)\right)-\bar{y} .
$$

Then (1.6) can be rewritten in the form

$$
\left\{\begin{array}{l}
\text { Minimize } \frac{1}{2} \iint_{Q} \rho^{2}|y|^{2}+\frac{1}{2} \iint_{\mathcal{O} \times(0, T)} \rho_{0}^{2}|f|^{2} \\
\text { Subject to }(y, f) \in \mathcal{Y} \times \mathcal{F}, M(y, f)=0
\end{array}\right.
$$

It is easy to check that $M$ is $C^{1}$ in $\mathcal{Y} \times \mathcal{F}$ and, in particular,

$$
M^{\prime}(y, f)(z, g)=z-H_{0}\left(g 1_{\mathcal{O} \times(0, T)}-F^{\prime}(y) z\right) \quad \forall(z, g) \in \mathcal{Y} \times \mathcal{F} .
$$


We also have

$$
M^{\prime}(y, f)^{*} \psi=\left(\psi+F^{\prime}(y) H_{0}^{*} \psi,-\left.\left(H_{0}^{*} \psi\right)\right|_{\mathcal{O} \times(0, T)}\right) \quad \forall \psi \in L^{2}(Q)
$$

and, since $H_{0}$ and $H_{0}^{*}$ are compact, as a consequence of Fredholm's Alternative Theorem, the rank $R\left(M^{\prime}(y, f)^{*}\right)$ is closed.

At this point, it is possible to apply the following result, usually known as Dubovitski-Milyoutin Formalism for extremal problems in Hilbert spaces (see [1, 12]):

Theorem 3.2. Let $\mathcal{H}$ and $\mathcal{E}$ be two Hilbert spaces. Let us assume that $I: \mathcal{H} \mapsto \mathbf{R}$ and $S: \mathcal{H} \mapsto \mathcal{E}$ are well-defined and $C^{1}$ and let us consider the extremal problem

$$
\left\{\begin{array}{l}
\text { Minimize } I(h) \\
\text { Subject to } h \in \mathcal{H}, S(h)=0 .
\end{array}\right.
$$

Let $\hat{h}$ be a solution to (3.5). Then, there exist $\lambda \in \mathbf{R}_{+}$and $\zeta \in N\left(S^{\prime}(\hat{h})\right)^{\perp}$, not both zero, such that

$$
-\lambda I^{\prime}(\hat{h})+\zeta=0
$$

Observation 3.3. A short explanation of (3.6) is the following: since $\hat{h}$ solves (3.5), there can be no descent direction at $\hat{h}$ admissible with respect to the constraint $S(h)=0$. In other words,

$$
\left\{d \in \mathcal{H}:\left(I^{\prime}(\hat{h}), d\right)_{\mathcal{H}}<0\right\} \text { and } N\left(S^{\prime}(\hat{h})\right) \text { are disjoint. }
$$

Accordingly, by duality, the algebraic sum of the associated conjugate sets contains the origin and this is precisely (3.6).

In what regards (3.4), in view of Theorem 3.2, we deduce that there exist $\lambda \in \mathbf{R}_{+}$and $(w, k) \in$ $N\left(M^{\prime}(y, f)\right)^{\perp}=R\left(M^{\prime}(y, f)^{*}\right)$ (not both zero) such that

$$
-\lambda\left(\rho^{2} y, \rho_{0}^{2} f\right)+(w, k)=0
$$

In other words,

$$
-\lambda\left(\rho^{2} y, \rho_{0}^{2} f\right)+\left(\psi+F^{\prime}(y) H_{0}^{*} \psi,-\left.\left(H_{0}^{*} \psi\right)\right|_{\mathcal{O} \times(0, T)}\right)=0
$$

for some $\psi \in L^{2}(Q)$.

Necessarily, one has $\lambda \neq 0$; otherwise, we would also have $\psi \equiv 0$ (from the unique continuation property) and then $(w, k)=(0,0)$. Hence, with $\phi=\frac{1}{\lambda} \psi$, we get:

$$
-\left(\rho^{2} y, \rho_{0}^{2} f\right)+\left(\phi+F^{\prime}(y) H_{0}^{*} \phi,-\left.\left(H_{0}^{*} \phi\right)\right|_{\mathcal{O} \times(0, T)}\right)=0,
$$

that is,

$$
f=-\left.\rho_{0}^{-2}\left(H_{0}^{*} \phi\right)\right|_{\mathcal{O} \times(0, T)}, \quad y=\rho^{-2}\left(\phi+F^{\prime}(y) H_{0}^{*} \phi\right) .
$$

Now, we take $p:=H_{0}^{*} \phi$, we see that $p \in \mathcal{P}$ and we find at once (3.2). 
Observe that (1.6) and (3.2) together yield

$$
L_{0}\left(\rho^{-2}\left(L_{0}^{*} p+F^{\prime}(y) p\right)\right)+F(y)+\rho_{0}^{-2} p 1_{\mathcal{O} \times(0, T)}=v 1_{\omega \times(0, T)} \text { in } Q
$$

and $y(\cdot, 0)=y_{0}$. Since $F(y)=F_{0}(y)\left(\rho^{-2}\left(L_{0}^{*} p+F^{\prime}(y) p\right)\right)$, this can also be written in the form

$$
L_{F_{0}(y)}\left(\rho^{-2}\left(L_{F^{\prime}(y)}^{*} p\right)+\rho_{0}^{-2} p 1_{\mathcal{O} \times(0, T)}=v 1_{\omega \times(0, T)} \text { in } Q\right.
$$

and $\left(\rho^{-2} L_{F^{\prime}(y)}^{*} p\right)(\cdot, 0)=y_{0}$. In other words, $p$ satisfies

$$
\iint_{Q}\left(\rho^{-2} L_{F^{\prime}(y)}^{*} p L_{F_{0}(y)}^{*} q+1_{\mathcal{O}} \rho_{0}^{-2} p q\right)=\iint_{\omega \times(0, T)} v q+\int_{\Omega} y_{0}(x) q(x, 0) \mathrm{d} x \quad \forall q \in \mathcal{P} .
$$

In particular, taking $q=p$, the following is obtained:

$$
\iint_{Q}\left(\rho^{-2} L_{F^{\prime}(y)}^{*} p L_{F_{0}(y)}^{*} p+1_{\mathcal{O}} \rho_{0}^{-2}|p|^{2}\right)=\iint_{\omega \times(0, T)} v p+\int_{\Omega} y_{0}(x) p(x, 0) \mathrm{d} x .
$$

Let us finally check that (3.3) holds.

Let us introduce $S:=\sup _{Q} \rho_{0} / \rho$. From (3.1), we know that $S<1 /(\sqrt{2} R)$. Consequently, $R^{2} S^{2} /\left(1-R^{2} S^{2}\right)<$ 1 and there exists $\beta$ satisfying

$$
\frac{1}{R} \frac{R^{2} S^{2}}{1-R^{2} S^{2}}<\beta<\frac{1}{R}
$$

From (3.7), we see that

$$
\begin{aligned}
m(0 ; p, p)= & \iint_{Q}\left(\rho^{-2}\left|L_{0}^{*} p\right|^{2}+1_{\mathcal{O}} \rho_{0}^{-2}|p|^{2}\right) \\
= & \iint_{\omega \times(0, T)} v p+\int_{\Omega} y_{0}(x) p(x, 0) \mathrm{d} x \\
& -\iint_{Q} \rho^{-2}\left(F^{\prime}(y) p L_{0}^{*} p+L_{0}^{*} p F_{0}(y) p+F^{\prime}(y) F_{0}(y)|p|^{2}\right) \\
\leq & C\|v\|_{\mathcal{U}}\left(\iint_{Q} \rho_{0}^{-2}|p|^{2}\right)^{1 / 2}+\left\|y_{0}\right\| \max _{[0, T / 2]}\|p(\cdot, t)\| \\
& +2 R \iint_{Q} \rho^{-2}\left|L_{0}^{*} p\right||p|+R^{2} \iint_{Q} \rho^{-2}|p|^{2} \\
\leq & C\left(\|v\|_{\mathcal{U}}+\left\|y_{0}\right\|\right) m(0 ; p, p)^{1 / 2}+\beta R \iint_{Q} \rho^{-2}\left|L_{0}^{*} p\right|^{2}+\left(R^{2}+\frac{R}{\beta}\right) \iint_{Q} \rho^{-2}|p|^{2} \\
\leq & C\left(\|v\|_{\mathcal{U}}+\left\|y_{0}\right\|\right) m(0 ; p, p)^{1 / 2}+\max \left(\beta R,\left(R^{2}+\frac{R}{\beta}\right) S^{2}\right) m(0 ; p, p) .
\end{aligned}
$$

Taking into account that $\beta R<1$ and $\left(R^{2}+R / \beta\right) S^{2}<1$, we deduce that

$$
m(0 ; p, p)^{1 / 2} \leq C\left(\|v\|_{\mathcal{U}}+\left\|y_{0}\right\|\right) .
$$

This proves the first part of (3.3). The second estimate in (3.3) is an immediate consequence.

This ends the proof. 
Proposition 3.4. Let us set

$$
\mathcal{G}:=\{(v, f): v \in \mathcal{U}, \quad f \in \Phi[v]\}
$$

where, for each leader $v \in \mathcal{U}, \Phi[v]$ denotes the set of the corresponding followers, i.e. the family of solutions to (1.6). Then $\mathcal{G}$ is weakly closed in $\mathcal{U} \times \mathcal{F}$ and the function $(v, f) \mapsto P(v ; f)$ is coercive and sequentially weakly lower semicontinuous.

Proof. Let $\left\{\left(v^{n}, f^{n}\right)\right\}$ be a sequence in $\mathcal{G}$, let the $y^{n}$ be the associated states and let us assume that $\left(v^{n}, f^{n}\right)$ converges weakly in $\mathcal{U} \times \mathcal{F}$ to some $(v, f)$. Then, it can be assumed that the $y^{n}$ converge strongly in $L^{2}(Q)$ to the state $y$ corresponding to $(v, f)$.

Let us check that $f$ solves (1.6). This will prove that $(v, f) \in \mathcal{G}$ and, accordingly, $\mathcal{G}$ is weakly closed. Indeed, if $f$ were not a solution to (1.6), there would exist $\hat{f} \in \mathcal{F}$ such that

$$
\iint_{Q} \rho^{2}|\hat{y}|^{2}+\iint_{\mathcal{O} \times(0, T)} \rho_{0}^{2}|\hat{f}|^{2}<\iint_{Q} \rho^{2}|y|^{2}+\iint_{\mathcal{O} \times(0, T)} \rho_{0}^{2}|f|^{2},
$$

where $\hat{y}$ is the state corresponding to $(v, \hat{f})$. Consequently, for $n$ large enough, we would also have

$$
\iint_{Q} \rho^{2}|\hat{y}|^{2}+\iint_{\mathcal{O} \times(0, T)} \rho_{0}^{2}|\hat{f}|^{2}<\iint_{Q} \rho^{2}\left|y^{n}\right|^{2}+\iint_{\mathcal{O} \times(0, T)} \rho_{0}^{2}\left|f^{n}\right|^{2}
$$

and also

$$
\iint_{Q} \rho^{2}\left|\hat{y}^{n}\right|^{2}+\left.\iint_{\mathcal{O} \times(0, T)} \rho_{0}^{2}\left|\hat{f}^{2}<\iint_{Q} \rho^{2}\right| y^{n}\right|^{2}+\iint_{\mathcal{O} \times(0, T)} \rho_{0}^{2}\left|f^{n}\right|^{2}
$$

where $\hat{y}^{n}$ is the state corresponding to $\left(v^{n}, \hat{f}\right)$. But this contradicts that $\left(v^{n}, f^{n}\right) \in \mathcal{G}$.

That $(v, f) \mapsto P(v ; f)$ is sequentially weakly lower semicontinuous is obvious. Let us finally see that it is coercive. Thus, let us assume that $\left(v^{n}, f^{n}\right) \in \mathcal{G}$ for all $n$ and $\left\|f^{n}\right\|_{\mathcal{F}} \rightarrow+\infty$. In view of Proposition 3.1, the couples $\left(v^{n}, f^{n}\right)$ must satisfy the second estimates in (3.3), whence $\left\|v^{n}\right\|_{\mathcal{U}} \rightarrow+\infty$ and, also, $P\left(v^{n} ; f^{n}\right) \rightarrow+\infty$.

This ends the proof.

Let us achieve the proof of Theorem 1.2. Again, the argument is classical.

Let $\left\{\left(v^{n}, f^{n}\right)\right\}$ be a minimizing sequence for (1.13). Then, $\left(v^{n}, f^{n}\right)$ are obviously uniformly bounded in $\mathcal{U} \times$ $\mathcal{F}$. Therefore, it can be assumed that $\left(v^{n}, f^{n}\right)$ converges weakly in this space to some $(\hat{v}, \hat{f}) \in \mathcal{G}$ and the corresponding states $y^{n}$ converge strongly in $L^{2}(Q)$ to the associated $\hat{y}$.

Since $(v, f) \mapsto P(v ; f)$ is sequentially weakly lower semicontinuous,

$$
P(\hat{v} ; \hat{f}) \leq \liminf _{n \rightarrow+\infty} P\left(v^{n} ; f^{n}\right)=\inf _{(v, f)} P(v ; f) .
$$

Consequently, $(\hat{v} ; \hat{f})$ solves $(1.13)$ and the proof is done.

Let us end this section with a characterization result:

Theorem 3.5. Let us assume that, in (1.2), $F: \mathbf{R} \mapsto \mathbf{R}$ is $C^{2}$, posseses bounded derivatives of order 1 and 2 and satisfies $F(0)=0$ and $y_{0} \in L^{2}(\Omega)$. Let $(\hat{v}, \hat{f})$ be a solution to (1.13). Then, the couple $(\hat{v}, \hat{f})$ must satisfy, 
together with the associated $\hat{y}, \hat{p}, \hat{\phi}$ and $\hat{\psi}$, the following optimality system:

$$
\begin{aligned}
& \begin{cases}\hat{y}_{t}-\Delta \hat{y}+F(\hat{y})=\hat{f} 1_{\mathcal{O}}+\hat{v} 1_{\omega} & \text { in } \quad Q \\
\hat{y}=0 & \text { on } \Sigma \\
\hat{y}(\cdot, 0)=y_{0} & \text { in } \quad \Omega\end{cases} \\
& \hat{f}=-\left.\rho_{0}^{-2} \hat{p}\right|_{\mathcal{O} \times(0, T)}, \quad \hat{y}=\rho^{-2} L_{F^{\prime}(\hat{y})}^{*} \hat{p}, \\
& \left\{\begin{array}{l}
\iint_{Q}\left(\rho^{-2} L_{F^{\prime}(\hat{y})}^{*} \hat{p} L_{F_{0}(\hat{y})}^{*} q+\rho_{0}^{-2} 1_{\mathcal{O}} \hat{p} q\right)=\iint_{\omega \times(0, T)} \hat{v} q+\int_{\Omega} y_{0}(x) q(x, 0) \mathrm{d} x \\
\forall q \in \mathcal{P}, \quad \hat{p} \in \mathcal{P} .
\end{array}\right. \\
& \begin{cases}-\hat{\phi}_{t}-\Delta \hat{\phi}+F^{\prime}(\hat{y}) \hat{\phi}=\alpha\left(\hat{y}-y_{d}\right) 1_{\omega_{d}}-F^{\prime}(\hat{y}) \hat{\psi}-\rho^{-2} F^{\prime \prime}(\hat{y}) \hat{p} L_{0}^{*} \hat{\psi} & \text { in } \quad Q \\
\hat{\phi}=0 & \text { on } \Sigma, \\
\hat{\phi}(\cdot, T)=0 & \text { in } \Omega\end{cases} \\
& \left\{\begin{array}{l}
\iint_{Q}\left(\rho^{-2} L_{F^{\prime}(\hat{y})}^{*} q L_{0}^{*} \hat{\psi}+\rho_{0}^{-2} 1_{\mathcal{O}} q \hat{\psi}\right)=-\iint_{\mathcal{O} \times(0, T)} \rho_{0}^{-2} \hat{\phi} q \\
\forall q \in \mathcal{P}, \quad \hat{\psi} \in \mathcal{P}
\end{array}\right. \\
& \hat{v}=-\left.\frac{1}{\mu} \rho_{0}^{-2}(\hat{\phi}+\hat{\psi})\right|_{\omega \times(0, T)} .
\end{aligned}
$$

Proof. We will deduce (3.8)-(3.13) as a consequence of the Dubovitskii-Milyoutin formalism applied to (1.8). In view of Proposition 3.1, we can reformulate (1.13) in the form

$$
\left\{\begin{array}{l}
\text { Minimize } P_{0}(y, v, f, p) \\
\text { Subject to }(y, v, f, p) \in \mathcal{X}, K(y, v, f, p)=(0,0,0)
\end{array}\right.
$$

where we have used the following notation

$$
\begin{gathered}
\mathcal{X}:=\mathcal{Y} \times \mathcal{U} \times \mathcal{F} \times \mathcal{P}, \\
P_{0}(y, v, f, p):=P(v ; f)=\frac{\alpha}{2} \iint_{\omega_{d} \times(0, T)}\left|y-y_{d}\right|^{2}+\frac{\mu}{2} \iint_{\omega \times(0, T)} \rho_{0}^{2}|v|^{2}, \\
K(y, v, f, p):=\left(y-H_{0}\left(v 1_{\omega}+f 1_{\mathcal{O}}-F(y)\right)-\bar{y}, y-\rho^{-2} L_{F^{\prime}(y)}^{*} p, f+\left.\rho_{0}^{-2} p\right|_{\mathcal{O} \times(0, T)}\right),
\end{gathered}
$$


we have introduced the linear compact operator $H_{0}: L^{2}(Q) \mapsto L^{2}(Q)$ with

$$
z=H_{0} h \Leftrightarrow \begin{cases}z_{t}-\Delta z=h & \text { in } Q, \\ z=0 & \text { on } \Sigma, \\ z(\cdot, 0)=0 & \text { in } \Omega\end{cases}
$$

and $\bar{y}$ is the unique solution to the uncontrolled problem

$$
\begin{cases}\bar{y}_{t}-\Delta \bar{y}=0 & \text { in } Q \\ \bar{y}=0 & \text { on } \Sigma \\ \bar{y}(\cdot, 0)=y_{0} & \text { in } \Omega .\end{cases}
$$

In view of the properties satisfied by $F$, the mapping $K: \mathcal{X} \mapsto L^{2}(Q) \times \mathcal{Y} \times \mathcal{F}$ is well defined and $C^{1}$, with

$$
\begin{aligned}
K^{\prime}(y, v, f, p)(z, w, g, q)= & \left(z-H_{0}\left(w 1_{\omega}+g 1_{\mathcal{O}}-F^{\prime}(y) z\right),\right. \\
& g+\left.\rho_{0}^{-2} q\right|_{\mathcal{O} \times(0, T)}, \\
& \left.z-\rho^{-2}\left(L_{F^{\prime}(y)}^{*} q+F^{\prime \prime}(y) z p\right)\right)
\end{aligned}
$$

for all $(y, v, f, p),(z, w, g, q) \in \mathcal{X}$. Accordingly, the adjoint $K^{\prime}(y, v, f, p)^{*}$ is given by

$$
\begin{aligned}
K^{\prime}(y, v, f, p)^{*}(\zeta, \beta, \eta)= & \left(\zeta+F^{\prime}(y) H_{0}^{*} \zeta+\beta-\rho^{-2} F^{\prime \prime}(y) p \beta,\right. \\
& -\left.\left(H_{0}^{*} \zeta\right)\right|_{\omega \times(0, T)}, \\
& \eta-\left.\left(H_{0}^{*} \zeta\right)\right|_{\mathcal{O} \times(0, T)} \\
& \left.\rho_{0}^{-2} \eta 1_{\mathcal{O}}-L_{F^{\prime}(y)}\left(\rho^{-2} \beta\right)\right)
\end{aligned}
$$

for any $(y, v, f, p) \in \mathcal{X}$ and any $(\zeta, \beta, \eta) \in L^{2}(Q) \times \mathcal{Y} \times \mathcal{F}$.

Let $(\hat{v}, \hat{f})$ be a solution to $(1.8)$, let $\hat{y}$ the associated state and let $\hat{p} \in \mathcal{P}$ be such that

$$
\hat{f}=-\left.\rho_{0}^{-2} \hat{p}\right|_{\mathcal{O} \times(0, T)}, \quad \hat{y}=\rho^{-2} L_{F^{\prime}(\hat{y})}^{*} \hat{p} .
$$

Then, $(\hat{y}, \hat{v}, \hat{f}, \hat{p})$ solves $(3.14)$.

It is not difficult to check that the ranks of $K^{\prime}(y, v, f, p)$ and $K^{\prime}(y, v, f, p)^{*}$ are closed. Consequently, we can apply Theorem 3.2 to (3.14): the cone of descent directions and the space of tangent directions at $(y, v, f, p)$ are disjoint and there exist multipliers $\lambda \in \mathbf{R}_{+}$and $(\zeta, \beta, \eta) \in L^{2}(Q) \times \mathcal{Y} \times \mathcal{F}$, not all zero, such that

$$
-\lambda\left(\alpha\left(\hat{y}-y_{d}\right) 1_{\omega_{d}}, \mu \rho_{0}^{2} \hat{v}, 0,0\right)+K^{\prime}(\hat{y}, \hat{v} \hat{f}, \hat{p})^{*}(\zeta, \beta, \eta)=(0,0,0,0) .
$$

Necessarily, $\lambda>0$. Indeed, if this is not the case, we must have $H_{0}^{*} \zeta=0$ in $\omega \times(0, T)$ and then $\zeta \equiv 0$ (as a consequence of unique continuation) and also $\eta=0$ and $\beta=0$. Hence, we can assume that $\lambda=1$ and this directly gives

$$
\begin{gathered}
\alpha\left(\hat{y}-y_{d}\right) 1_{\omega_{d}}=\zeta+F^{\prime}(y) H_{0}^{*} \zeta+\beta-\rho^{-2} F^{\prime \prime}(y) p \beta, \quad \hat{v}=-\left.\frac{1}{\mu} \rho_{0}^{-2} H_{0}^{*} \zeta\right|_{\omega \times(0, T)}, \\
\eta=\left.H_{0}^{*} \zeta\right|_{\mathcal{O} \times(0, T)}, \quad L_{F^{\prime}(y)}\left(\rho^{-2} \beta\right)-\rho_{0}^{-2} \eta 1_{\mathcal{O}}=0 .
\end{gathered}
$$


Let us set $\hat{\phi}:=H_{0}^{*}(\zeta+\beta)$ and $\hat{\psi}:=-H_{0}^{*} \beta$. Then, it is clear that $H_{0}^{*} \zeta=\hat{\phi}+\hat{\psi}$ and $\hat{v}, \hat{\phi}$ and $\hat{\psi}$ satisfy (3.12)(3.13).

This ends the proof.

For example, the assumptions in Theorem 3.5 are satisfied by any $F$ of the form

$$
F(s)=\frac{p(s)}{q(s)} s \quad \forall s \in \mathbf{R},
$$

where $p$ and $q$ are polynomial functions respectively of degree $n$ and $m$ with $n \leq m$ and $q(s) \neq 0$ for all $s \in \mathbf{R}$.

\section{LEADERS WITH CONSTRAINTS}

Let us begin this section by sketching the proof of Theorem 1.3.

In fact, it is not very different from the proof of Theorem 1.1. It is again a consequence of Propositions 2.3 and 2.5. Indeed, the properties of the mapping $v \mapsto f[v]$ and the functional in (1.4) guarantee that $J$ possesses exactly one minimizer in $\mathcal{U}_{a d}$.

We also have the following:

Theorem 4.1. The unique minimizer $\hat{v}$ of $J$ in $\mathcal{U}_{\text {ad }}$ and the associated $\hat{p}, \hat{y}, \hat{\phi}$ and $\hat{\psi}$ satisfy (2.7)-(2.11) together with

$$
\hat{v}=P_{a d}\left(-\left.\frac{1}{\mu} \rho_{0}^{-2}(\hat{\psi}+\hat{\phi})\right|_{\mathcal{O} \times(0, T)}\right)
$$

where $P_{a d}: \mathcal{U} \mapsto \mathcal{U}_{a d}$ is the usual orthogonal projector.

Again, the proof is similar to the proof of Theorem 2.6. It suffices to notice that the unique minimizer in (1.8) subject to the constraint $v \in \mathcal{U}_{a d}$ must satisfy

$$
\left.\frac{\mathrm{d}}{\mathrm{d} \varepsilon} J(\hat{v}+\varepsilon(v-\hat{v}))\right|_{\varepsilon=0} \geq 0 \quad \forall v \in \mathcal{U}_{a d}
$$

Taking into account the definitions of $\hat{\phi}$ and $\hat{\psi}$, we readily see that this is equivalent to (4.1).

Observation 4.2. It makes sense to try to prove hierarchic control results when we impose a priori constraints on the leaders and the followers. Thus, let $\alpha, \beta>0$ be given and let us introduce the sets

$$
\mathcal{U}_{\alpha}:=\left\{v \in \mathcal{U}:\|v\|_{\mathcal{U}} \leq \alpha\right\} \text { and } \mathcal{F}_{\beta}:=\left\{f \in \mathcal{F}:\|f\|_{\mathcal{F}} \leq \beta\right\}
$$

For every $v \in \mathcal{U}_{\alpha}$, there exists a unique associated follower $f[v] \in \mathcal{F}$. Furthermore, in view of (2.5), one has

$$
\|f[v]\|_{\mathcal{F}} \leq C\left(\alpha+\left\|y_{0}\right\|\right)
$$

where $C$ only depends on $\Omega, \mathcal{O}, \omega, T$ and $a$. Consequently, if $\beta>C \alpha$ and the initial state satisfies $\left\|y_{0}\right\| \leq$ $\beta / C-\alpha$, we can find leaders $v \in \mathcal{U}_{\alpha}$ and associated followers $f[v] \in \mathcal{F}_{\beta}$ such that the corresponding states satisfy (1.7).

\section{Some ADDitional COMments And OPEN QUESTiOnS}

This section is devoted to discuss some extensions and variants of the problems analyzed above. We will consider only states governed by linear PDEs. Of course, similar nonlinear problems are interesting and deserve 
attention but their study unfortunately requires some technicalities that are out the scope of this work. They will be treated in a forthcoming paper.

\subsection{Multi-objective hierarchical problems and Pareto equilibria leaders}

Let $\omega_{1}, \omega_{2}$ and $\mathcal{O}$ be three non-empty mutually disjoint open subsets of $\Omega$ and let us consider the controlled system

$$
\begin{cases}y_{t}-\Delta y+a(x, t) y=f 1_{\mathcal{O}}+v_{1} 1_{\omega_{1}}+v_{2} 1_{\omega_{2}} & \text { in } Q \\ y=0 & \text { on } \Sigma, \\ y(\cdot, 0)=y_{0} & \text { in } \Omega\end{cases}
$$

where again $a \in L^{\infty}(Q)$ and $y_{0} \in L^{2}(\Omega)$. We will use the spaces $\mathcal{Y}$ and $\mathcal{F}$ defined in (1.5) and also the spaces

$$
\mathcal{U}_{i}:=\left\{v: \rho_{0} v \in L^{2}\left(\omega_{i} \times(0, T)\right)\right\}, \quad i=1,2 .
$$

Let the sets $\omega_{d, i} \subset \Omega$ be non-empty and open and let the functions $y_{d, i} \in L^{2}\left(\omega_{d, i} \times(0, T)\right)$ be given. We will consider the secondary functional

$$
S\left(v_{1}, v_{2} ; f\right):=\frac{1}{2} \iint_{Q} \rho^{2}|y|^{2}+\frac{1}{2} \iint_{\mathcal{O} \times(0, T)} \rho_{0}^{2}|f|^{2}
$$

and the main functionals

$$
P_{i}\left(v_{1}, v_{2} ; f\right):=\frac{\alpha_{i}}{2} \iint_{\omega_{d, i} \times(0, T)}\left|y-y_{d, i}\right|^{2}+\frac{\mu_{i}}{2} \iint_{\omega_{i} \times(0, T)} \rho_{0}^{2}\left|v_{i}\right|^{2},
$$

where the $\alpha_{i}, \mu_{i}>0$ and $\alpha_{i}+\mu_{i}=1$ for $i=1,2$.

The Pareto hierarchical control process for (5.1)-(5.3) is the following:

1. We associate to each leader couple $\left(v_{1}, v_{2}\right) \in \mathcal{U}_{1} \times \mathcal{U}_{2}$ the unique solution $f\left[v_{1}, v_{2}\right]$ to the extremal problem

$$
\left\{\begin{array}{l}
\text { Minimize } S\left(v_{1}, v_{2} ; f\right) \\
\text { subject to } f \in \mathcal{F}
\end{array}\right.
$$

Observe that the corresponding state $y$ must necessarily satisfy $y(\cdot, T)=0$. In the sequel, we set $G_{i}\left(v_{1}, v_{2}\right):=P_{i}\left(v_{1}, v_{2} ; f\left[v_{1}, v_{2}\right]\right)$ for all $\left(v_{1}, v_{2}\right)$.

2. Then, we look for a Pareto equilibrium $\left(v_{1}, v_{2}\right)$ in $\mathcal{U}_{1} \times \mathcal{U}_{2}$ for the functionals $G_{1}$ and $G_{2}$. By definition, this means that the following properties are satisfied:

$$
\begin{cases}\left(u_{1}, u_{2}\right) \in \mathcal{U}_{1} \times \mathcal{U}_{2}, & G_{1}\left(u_{1}, u_{2}\right)<G_{1}\left(v_{1}, v_{2}\right) \Rightarrow G_{2}\left(u_{1}, u_{2}\right)>G_{2}\left(v_{1}, v_{2}\right) \\ \left(u_{1}, u_{2}\right) \in \mathcal{U}_{1} \times \mathcal{U}_{2}, & G_{2}\left(u_{1}, u_{2}\right)<G_{2}\left(v_{1}, v_{2}\right) \Rightarrow G_{1}\left(u_{1}, u_{2}\right)>G_{1}\left(v_{1}, v_{2}\right)\end{cases}
$$

see $[18]$.

Arguing as in the proof of Proposition 2.3, it is not difficult to check that, for each $\left(v_{1}, v_{2}\right) \in \mathcal{U}_{1} \times \mathcal{U}_{2}$, there exists exactly one solution $f\left[v_{1}, v_{2}\right]$ to $(5.4)$ furthermore satisfying

$$
f\left[v_{1}, v_{2}\right]=-\left.\rho_{0}^{-2} p\right|_{\mathcal{O}}, \quad y=\rho^{-2} L_{a}^{*}(p)
$$


where $p$ is the unique solution to the linear problem

$$
\left\{\begin{array}{l}
m(a ; p, q)=\iint_{Q}\left(v_{1} 1_{\omega_{1}}+v_{2} 1_{\omega_{2}}\right) q+\int_{\Omega} y_{0}(x) q(x, 0) \mathrm{d} x \\
\forall q \in \mathcal{P}, \quad p \in \mathcal{P} .
\end{array}\right.
$$

The functionals $G_{i}: \mathcal{U}_{1} \times \mathcal{U}_{2} \mapsto \mathbf{R}$ are well defined, strictly convex and $C^{1}$. Consequently, it can be deduced from Lagrange's Multipliers Theorem that, if $\left(v_{1}, v_{2}\right)$ is an associated Pareto equilibrium, there exist $\lambda \in[0,1]$ such that

$$
\lambda G_{1}^{\prime}\left(v_{1}, v_{2}\right)+(1-\lambda) G_{2}^{\prime}\left(v_{1}, v_{2}\right)=0 .
$$

Also, if (5.6) is satisfied for some $\lambda \in[0,1]$, then $\left(v_{1}, v_{2}\right)$ is necessarily the unique minimizer of $\lambda G_{1}+(1-\lambda) G_{2}$ and, consequently, $\left(v_{1}, v_{2}\right)$ is a Pareto equilibrium for $G_{1}$ and $G_{2}$.

Since for any $\lambda \in(0,1)$ the functional $\lambda G_{1}+(1-\lambda) G_{2}$ is coercive, one has the following:

Theorem 5.1. There exists a family $\left\{\left(v_{1, \lambda}, v_{2, \lambda}\right)\right\}_{\lambda \in(0,1)}$ of Pareto equilibria for $G_{1}$ and $G_{2}$. For each $\lambda$, $\left(v_{1, \lambda}, v_{2, \lambda}\right)$ is the unique minimizer of $\lambda G_{1}+(1-\lambda) G_{2}$ in $\mathcal{U}_{1} \times \mathcal{U}_{2}$ and, accordingly, the unique solution to (5.6).

Arguing as in the proof of Theorem 2.6, it is possible to deduce that, for each $\lambda$, the couple $\left(v_{1, \lambda}, v_{2, \lambda}\right)$ must solve, together with the associated state $y^{\lambda}$ and some $p^{\lambda}, \phi^{\lambda}$ and $\psi^{\lambda}$, the following optimality system:

$$
\begin{aligned}
& \begin{cases}y_{t}^{\lambda}-\Delta y^{\lambda}+a(x, t) y^{\lambda}=f\left[v_{1, \lambda}, v_{2, \lambda}\right] 1_{\mathcal{O}}+v_{1, \lambda} 1_{\omega_{1}}+v_{2, \lambda} 1_{\omega_{2}} & \text { in } Q, \\
y^{\lambda}=0 & \text { on } \Sigma, \\
y^{\lambda}(\cdot, 0)=y_{0} & \text { in } \Omega,\end{cases} \\
& f\left[v_{1, \lambda}, v_{2, \lambda}\right]=-\rho_{0}^{-2} p^{\lambda} 1_{\mathcal{O}}, \quad y^{\lambda}=\rho^{2} L_{a}^{*} p^{\lambda}, \\
& \left\{\begin{array}{l}
m\left(a ; p^{\lambda}, q\right)=\iint_{Q}\left(v_{1, \lambda} 1_{\omega_{1}}+v_{2, \lambda} 1_{\omega_{2}}\right) q+\int_{\Omega} y_{0}(x) q(x, 0) \mathrm{d} x \\
\forall q \in \mathcal{P}, \quad p^{\lambda} \in \mathcal{P},
\end{array}\right. \\
& v_{1, \lambda}=-\frac{1}{\lambda \mu_{1}} \rho_{0}^{-2}\left(\left(\psi^{\lambda}+\phi^{\lambda}\right) 1_{\omega_{1}}\right), \quad v_{2, \lambda}=-\frac{1}{(1-\lambda) \mu_{2}} \rho_{0}^{-2}\left(\left(\psi^{\lambda}+\phi^{\lambda}\right) 1_{\omega_{2}}\right), \\
& \begin{cases}-\phi_{t}^{\lambda}-\Delta \phi^{\lambda}+a(x, t) \phi^{\lambda}=\lambda \alpha_{1}\left(y^{\lambda}-y_{d, 1}\right) 1_{\omega_{d, 1}}+(1-\lambda) \alpha_{2}\left(y^{\lambda}-y_{d, 2}\right) 1_{\omega_{d, 2}} & \text { in } Q, \\
\phi^{\lambda}=0 & \text { on } \Sigma, \\
\phi^{\lambda}(\cdot, T)=0 & \text { in } \Omega,\end{cases} \\
& \left\{\begin{array}{l}
m\left(a ; q, \psi^{\lambda}\right)=\iint_{\mathcal{O} \times(0, T)} \phi^{\lambda} q \\
\forall q \in \mathcal{P}, \quad \psi^{\lambda} \in \mathcal{P} .
\end{array}\right.
\end{aligned}
$$

The computation of the Pareto equilibria $\left(v_{1, \lambda}, v_{2, \lambda}\right)$ can be carried out by solving the optimality system. To this purpose, for any fixed $\lambda \in(0,1)$, a natural fixed-point algorithm is the following: 
a) Fix $\left(v_{1}^{0}, v_{2}^{0}\right) \in \mathcal{U}_{1} \times \mathcal{U}_{2}$.

b) Then, for any given $n \geq 0$ and $\left(v_{1}^{n}, v_{2}^{n}\right)$, compute $p^{n}, f^{n}$ and $y^{n}$ from (5.9) and (5.8), compute $\phi^{n}$ and $\psi^{n}$ from (5.11) and (5.12) and finally compute $\left(v_{1}^{n+1}, v_{2}^{n+1}\right)$ from (5.10).

It is not difficult to check that, at least when $\alpha_{1} / \mu_{1}$ and $\alpha_{2} / \mu_{2}$ are small enough, these iterates converge strongly in $\mathcal{U}_{1} \times \mathcal{U}_{2}$ to a Pareto equilibrium.

\subsection{Boundary followers}

In this section, we will deal with a hierarchical control problem where the follower acts on a part of the boundary.

Thus, let $\omega \subset \Omega$ be a non-empty open set, let $\gamma$ be a relatively open subset of the boundary $\partial \Omega$ and let us consider the state system

$$
\begin{cases}y_{t}-\Delta y+a(x, t) y=v 1_{\omega} & \text { in } \quad Q, \\ y=f 1_{\gamma} & \text { on } \Sigma, \\ y(\cdot, 0)=y_{0} & \text { in } \Omega,\end{cases}
$$

where (again) $a \in L^{\infty}(Q)$ and $y_{0} \in L^{2}(\Omega)$.

Let the function $\tilde{\eta}_{0}$ be such that

$$
\left\{\begin{array}{l}
\tilde{\eta}_{0} \in C^{2}(\bar{\Omega}), \quad \tilde{\eta}_{0} \geq 0 \text { and } \nabla \tilde{\eta}_{0} \neq 0 \text { in } \Omega \\
\frac{\partial \tilde{\eta}_{0}}{\partial \nu} \leq 0 \text { on } \partial \Omega \backslash \gamma
\end{array}\right.
$$

let $\tilde{\sigma}$ and $\tilde{\xi}$ be the analog of the functions $\sigma$ and $\xi$ in (1.11) with $\eta$ replaced by $\tilde{\eta}_{0}$ and let us introduce the weights $\varrho=e^{s \tilde{\sigma}}, \varrho_{0}=(s \tilde{\xi})^{-3 / 2} \lambda^{-2} \varrho, \varrho_{1}=(s \tilde{\xi})^{-1 / 2} \lambda^{-1} \varrho, \varrho_{2}=(s \tilde{\xi})^{-1 / 2} \varrho$ and $\varrho_{3}=(s \tilde{\xi})^{1 / 2} \varrho$.

With this in mind, let us consider the secondary and main functionals

$$
\begin{aligned}
& S^{*}(v ; f):=\frac{1}{2} \iint_{Q} \varrho^{2}|y|^{2}+\frac{1}{2} \iint_{\gamma \times(0, T)} \varrho_{2}^{2}|f|^{2} \mathrm{~d} \Gamma \mathrm{d} t \\
& P^{*}(v ; f):=\frac{\alpha}{2} \iint_{\omega_{d} \times(0, T)}\left|y-y_{d}\right|^{2}+\frac{\mu}{2} \iint_{\omega \times(0, T)} \varrho_{0}^{2}|v|^{2}
\end{aligned}
$$

and the spaces

$$
\begin{gathered}
\mathcal{U}^{*}:=\left\{v: \varrho_{0} v \in L^{2}(\omega \times(0, T))\right\} \\
\mathcal{Y}^{*}:=\left\{y: \varrho y \in L^{2}(Q)\right\} \text { and } \mathcal{F}^{*}:=\left\{f: \varrho_{2} f \in L^{2}(\gamma \times(0, T))\right\}
\end{gathered}
$$

As before, to each leader $v \in \mathcal{U}^{*}$ we associate the unique solution $f[v]$ to the extremal problem

$$
\left\{\begin{array}{l}
\text { Minimize } S^{*}(v ; f) \\
\text { subject to } f \in \mathcal{F}^{*}
\end{array}\right.
$$

Then, we consider the functional $v \mapsto P^{*}(v ; f[v])$ and we try to find $\hat{v}$ satisfying

$$
P^{*}(\hat{v}, f[\hat{v}]) \leq P^{*}(v, f[v]) \quad \forall v \in \mathcal{U}^{*}, \quad \hat{v} \in \mathcal{U}^{*} .
$$


In this case, we must define in the space $\mathcal{P}_{0}$ the bilinear form

$$
b(a ; p, \tilde{p})=\iint_{Q} \varrho^{-2} L_{a}^{*} p L_{a}^{*} q+\iint_{\gamma \times(0, T)} \varrho_{2}^{-2} \frac{\partial p}{\partial \nu} \frac{\partial q}{\partial \nu} \mathrm{d} \Gamma \mathrm{d} t .
$$

In view of the unique continuation property, $b(a ; \cdot, \cdot)$ is a norm in $\mathcal{P}_{0}$. Let us denote by $\mathcal{B}$ the corresponding Hilbert completion. Then, we can use a new Carleman inequality involving the values on the boundary of the normal derivatives. It is given in the following result:

Theorem 5.2. There exist positive constants $\lambda_{1}, s_{1}$ and $C_{1}$, only depending on $\Omega, \gamma$ and $T$, such that, if we take $\lambda=\lambda_{1}$ and $s=s_{1}$, any $p \in \mathcal{B}$ satisfies

$$
\iint_{Q}\left[\varrho_{3}^{-2}\left(\left|p_{t}\right|^{2}+|\Delta p|^{2}\right)+\varrho_{1}^{-2}|\nabla p|^{2}+\varrho_{0}^{-2}|p|^{2}\right] \leq C_{1} b(0 ; p, p) .
$$

Furthermore, $\lambda_{1}$ and $s_{1}$ can be found arbitrarily large.

In the remainder of this section, we take $\lambda=\lambda_{1}$ and $s=s_{1}$. Then, as in Section 2, we can find positive constants $K_{0}$ and $K_{1}$, only depending on $\Omega, \gamma, T$ and $\|a\|_{L^{\infty}(Q)}$, such that

$$
K_{0} b(0 ; p, p) \leq b(a ; p, p) \leq K_{1} b(0 ; p, p) \quad \forall p \in \mathcal{B} .
$$

As before, for each $v \in \mathcal{U}^{*}$, there exists exactly one solution $f[v]$ to (5.13). Furthermore, the follower $f[v]$ and the associated state $y$ satisfy

$$
f[v]=\left.\varrho_{2}^{-2} \frac{\partial p}{\partial \nu}\right|_{\gamma \times(0, T)}, \quad y=\varrho^{-2} L_{a}^{*} p
$$

where $p$ is the unique solution to the problem

$$
\left\{\begin{array}{l}
b(a ; p, q)=\iint_{\omega \times(0, T)} v q+\int_{\Omega} y_{0}(x) q(x, 0) \mathrm{d} x \\
\forall q \in \mathcal{B}, \quad p \in \mathcal{B} .
\end{array}\right.
$$

As in Section 2, it can be deduced that there exists a unique leader $\hat{v}$ satisfying (5.15). We also have that $\hat{v}$ satisfies, together with the associated state $\hat{y}$ and some $\hat{p}, \hat{\phi}$ and $\hat{\psi}$, the following:

$$
\begin{gathered}
\begin{cases}\hat{y}_{t}-\Delta \hat{y}+a(x, t) \hat{y}=\hat{v} 1_{\omega} & \text { in } \Omega \\
\hat{y}=f[\hat{v}] 1_{\gamma} & \text { on } \Sigma, \\
\hat{y}(\cdot, 0)=y_{0} & \text { in } \Omega,\end{cases} \\
f[\hat{v}]=\left.\varrho_{2}^{-2} \frac{\partial \hat{p}}{\partial \nu}\right|_{\gamma}, \quad \hat{y}=\varrho^{-2} L_{a}^{*} \hat{p}, \\
\left\{\begin{array}{l}
b(a ; \hat{p}, q)=\iint_{\omega \times(0, T)} \hat{v} q+\iint_{\Omega} y_{0}(x) q(x, 0) \mathrm{d} x \\
\forall q \in \mathcal{B}, \quad \hat{p} \in \mathcal{B},
\end{array}\right.
\end{gathered}
$$




$$
\begin{aligned}
& \begin{cases}-\hat{\phi}_{t}-\Delta \hat{\phi}+a(x, t) \hat{\phi}=\alpha\left(\hat{y}-y_{d}\right) 1_{\omega_{d}} & \text { in } \Omega, \\
\hat{\phi}=0 & \text { on } \Sigma, \\
\hat{\phi}(\cdot, T)=0 & \text { in } \Omega,\end{cases} \\
& \hat{v}=-\left.\frac{1}{\mu} \varrho_{0}^{-2}(\hat{\phi}+\hat{\psi})\right|_{\omega \times(0, T)}, \\
& \left\{\begin{array}{l}
b(a ; q, \hat{\psi})=\iint_{\gamma \times(0, T)} \varrho_{2}^{-2} \frac{\partial \hat{\phi}}{\partial \nu} \frac{\partial q}{\partial \nu} \mathrm{d} \Gamma \mathrm{d} t, \\
\forall q \in \mathcal{B}, \quad \hat{\psi} \in \mathcal{B} .
\end{array}\right.
\end{aligned}
$$

\subsection{Boundary leaders and followers}

Let $\gamma$ and $\sigma$ disjoint open subsets of the boundary $\partial \Omega$ and, again, let $\omega_{d} \subset \Omega$ be a non-empty open set where an objective function $y_{d}$ is defined. Let us consider the state system

$$
\begin{cases}y_{t}-\Delta y+a(x, t) y=0 & \text { in } \Omega, \\ y=f 1_{\gamma}+v 1_{\sigma} & \text { in } \Sigma, \\ y(\cdot, 0)=y_{0} & \text { in } \Omega,\end{cases}
$$

where we find a boundary leader $v$ and a boundary follower $f$, respectively acting on $\gamma \times(0, T)$ and $\sigma \times(0, T)$.

For the analysis of this problem, we need the weight functions defined in Section 5.2 together with the following $x$-independent weight function:

$$
\zeta(t):=\max _{x \in \bar{\Omega}} \varrho_{2}(t, x)
$$

This way, we can use the secondary functional $S^{*}$, the main functional

$$
\tilde{P}(v ; f):=\frac{\alpha}{2} \iint_{\omega_{d} \times(0, T)}\left|y-y_{d}\right|^{2}+\frac{\mu}{2} \iint_{\sigma \times(0, T)} \zeta^{2}|v|^{2} \mathrm{~d} \Gamma \mathrm{d} t
$$

the space

$$
\tilde{\mathcal{U}}:=\left\{v: \zeta v \in L^{2}(\sigma \times(0, T))\right\}
$$

and the spaces $\mathcal{F}^{*}$ and $\mathcal{Y}^{*}$ and we can prove results similar to those above.

More precisely, to each leader $v \in \tilde{\mathcal{U}}$, we can associate the follower $f[v] \in \mathcal{F}^{*}$, the unique solution to the secondary extremal problem (5.14). One has

$$
f[v]=\left.\varrho_{2}^{-2} \frac{\partial p}{\partial \nu}\right|_{\gamma}, \quad y=\varrho^{-2} L_{a}^{*} p,
$$

where $p$ is the solution to the problem

$$
\left\{\begin{array}{l}
b(a ; p, q)=\iint_{\sigma \times(0, T)} v \frac{\partial q}{\partial \nu} \mathrm{d} \Gamma \mathrm{d} t+\iint_{Q} y_{0}(x) q(x, 0) \mathrm{d} x \\
\forall p \in \mathcal{B}, \quad p \in \mathcal{B} .
\end{array}\right.
$$


On the oher hand, the functional $v \mapsto \tilde{P}(v ; f[v])$ possesses exactly one minimizer $\hat{v}$ in $\tilde{\mathcal{U}}$. It satisfies, together with the associated state $\hat{y}$ and some $\hat{p}, \hat{\phi}$ and $\hat{\psi}$, the following optimality system:

$$
\begin{aligned}
& \begin{cases}\hat{y}_{t}-\Delta \hat{y}+a(x, t) \hat{y}=\hat{v} 1_{\omega} & \text { in } \quad \Omega \\
\hat{y}=f[\hat{v}] 1_{\gamma}+\hat{v} 1_{\sigma} & \text { on } \Sigma, \\
\hat{y}(\cdot, 0)=y_{0} & \text { in } \Omega,\end{cases} \\
& f[\hat{v}]=\left.\varrho_{2}^{-2} \frac{\partial \hat{p}}{\partial \nu}\right|_{\gamma \times(0, T)}, \quad \hat{y}=\varrho^{-2} L_{a}^{*} \hat{p} \\
& \left\{\begin{array}{l}
b(a ; \hat{p}, q)=\iint_{\sigma \times(0, T)} \hat{v} \frac{\partial q}{\partial \nu} \mathrm{d} \Gamma \mathrm{d} t+\iint_{\Omega} y_{0}(x) q(x, 0) \mathrm{d} x \\
\forall q \in \mathcal{B}, \quad \hat{p} \in \mathcal{B},
\end{array}\right. \\
& \begin{cases}-\hat{\phi}_{t}-\Delta \hat{\phi}+a(x, t) \hat{\phi}=\alpha\left(\hat{y}-y_{d}\right) 1_{\omega_{d}} & \text { in } \Omega, \\
\hat{\phi}=0 & \text { on } \Sigma, \\
\hat{\phi}(\cdot, T)=0 & \text { in } \Omega,\end{cases} \\
& \hat{v}=-\left.\frac{1}{\mu} \beta^{-2}\left(\frac{\partial \hat{\phi}}{\partial \nu}+\frac{\partial \hat{\psi}}{\partial \nu}\right)\right|_{\sigma \times(0, T)}, \\
& \left\{\begin{array}{l}
b(a ; q, \hat{\psi})=\iint_{\gamma \times(0, T)} \varrho_{2}^{-2} \frac{\partial \hat{\phi}}{\partial \nu} \frac{\partial q}{\partial \nu} \mathrm{d} \Gamma \mathrm{d} t \\
\forall q \in \mathcal{B}, \quad \hat{\psi} \in \mathcal{B} .
\end{array}\right.
\end{aligned}
$$

\subsection{Other parabolic PDEs and systems}

The results in Sections 2 and 3 can be extended to cover other more general situations. Thus, let us first consider systems of the form

$$
\begin{cases}M_{a} y=f 1_{\mathcal{O}}+v 1_{\omega} & \text { in } \quad Q \\ y=0 & \text { on } \Sigma \\ y(\cdot, 0)=y_{0} & \text { in } \Omega\end{cases}
$$

and

$$
\begin{cases}M_{a} y+F(y)=f 1_{\mathcal{O}}+v 1_{\omega} & \text { in } \quad Q \\ y=0 & \text { on } \Sigma \\ y(\cdot, 0)=y_{0} & \text { in } \Omega\end{cases}
$$

where

$$
M_{a} y:=y_{t}-\sum_{i, j=1}^{N} \partial_{j}\left(a_{i, j}(x, t) \partial_{i} y\right)+\sum_{i=1}^{N} b_{i}(x, t) \partial_{i} y+a(x, t) y
$$


the $a_{i, j} \in W^{1, \infty}(Q)$ are uniformly elliptic, the $b_{i} \in L^{\infty}(Q), a \in L^{\infty}(Q)$ and $F \in W^{1, \infty}(\mathbf{R})$.

It is known that Theorem 2.1 holds for the modified bilinear form $\tilde{m}(a ; \cdot, \cdot)$, given by

$$
\tilde{m}(a ; p, q):=\iint_{Q}\left(\rho^{-2} M_{a}^{*} p M_{a}^{*} q+1_{\mathcal{O}} \rho_{0}^{-2} p q\right)
$$

where we have used the notation

$$
M_{a}^{*} p=-p_{t}-\sum_{i, j=1}^{N} \partial_{i}\left(a_{i, j}(x, t) \partial_{j} p\right)-\sum_{i=1}^{N} \partial_{i}\left(b_{i}(x, t) p\right)+a(x, t) p
$$

see [11]. Also, it can be proved that Theorem 1.1 and Theorem 1.2 hold when the state system is respectively given by (5.27) and (5.28).

Now, let us consider the Stokes system

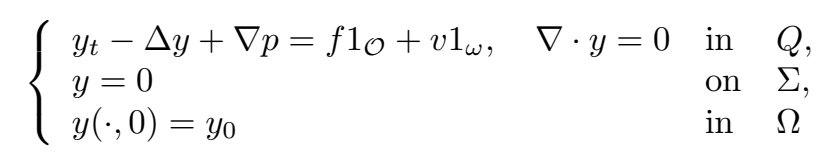

and the corresponding adjoint

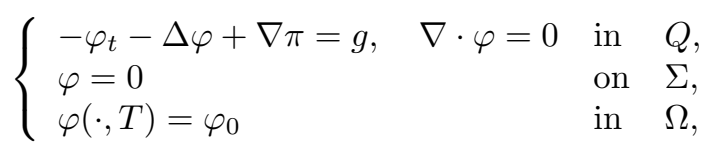

where $g \in L^{2}(Q)^{N}$ and $\varphi_{0} \in L^{2}(\Omega)^{N}, \nabla \cdot \varphi_{0}=0$ in $\Omega$ and the normal trace $\varphi \cdot n$ vanishes on $\partial \Omega$.

Then, a Carleman inequality of the kind (2.1) can be established for the solutions to the adjoint systems (5.30). More precisely, with appropriate new definitions of the weights $\rho_{0}, \rho_{1}, \ldots$, one has

$$
\iint_{Q}\left[\rho_{2}^{-2}\left(\left|\varphi_{t}\right|^{2}+|\Delta \varphi|^{2}\right)+\rho_{1}^{-2}|\nabla \varphi|^{2}+\rho_{0}^{-2}|\varphi|^{2}\right] \leq C \hat{m}(0 ;(\varphi, \pi),(\varphi, \pi))
$$

for all $(\varphi, \pi)$, where we have set

$$
\hat{m}\left(0 ;(\varphi, \pi),\left(\varphi^{\prime}, \pi^{\prime}\right)\right):=\iint_{Q}\left(\rho^{-2}\left(L_{0}^{*} \varphi+\nabla \pi\right)\left(L_{0}^{*} \varphi^{\prime}+\nabla \pi^{\prime}\right)+1_{\mathcal{O}} \rho_{0}^{-2} \varphi \varphi^{\prime}\right)
$$

and $C$ only depends on $\Omega, \mathcal{O}$ and $T$; see [14].

As a consequence, proceeding as in Section 2, we get the following analog of Theorem 1.1:

Theorem 5.3. Let us assume that $y_{0} \in L^{2}(\Omega)^{N}, \nabla \cdot y_{0}=0$ in $\Omega$ and $y \cdot n=0$ on $\partial \Omega$. Let us consider the problems (1.6) and (1.8), where for each $f \in \mathcal{F}^{N}$ and each $v \in \mathcal{U}^{N}, y$ is, together with some $p$, the solution to the corresponding system (5.29).

1. For every $v \in \mathcal{U}^{N}$, there exists exactly one solution $f[v]$ to (1.6).

2. Let us set $J(v):=P(v ; f[v])$. There exists exactly one minimizer $\hat{v}$ of $J$ in $\mathcal{U}^{N}$ and, consequently, one associated follower $f[\hat{v}]$ such that (1.7) holds. 
Finally, let us consider the $n \times n$ parabolic system

$$
\begin{cases}y_{t}-D \Delta y+A y=B f 1_{\mathcal{O}}+R v 1_{\omega} & \text { in } Q, \\ y=0 & \text { on } \Sigma, \\ y(\cdot, 0)=y_{0} & \text { in } \Omega,\end{cases}
$$

where $D \in \mathcal{L}\left(\mathbf{R}^{n} ; \mathbf{R}^{n}\right)$ is diagonalizable and definite positive, $A \in \mathcal{L}\left(\mathbf{R}^{n} ; \mathbf{R}^{n}\right), B \in L\left(\mathbf{R}^{m} ; \mathbf{R}^{n}\right)$ and $R \in$ $L\left(\mathbf{R}^{k} ; \mathbf{R}^{n}\right)$. Here, the integers $m, k \leq n$ and the controls are $f=\left(f_{1}, \ldots, f_{m}\right)$ and $v=\left(v_{1}, \ldots, v_{k}\right)$.

For any fixed $v \in \mathcal{U}^{k},(5.31)$ is null-controllable with controls in $\mathcal{F}^{m}$ if and only if

$$
\text { Rank }\left[B\left|\left(-\lambda_{p} D+A\right) B\right| \cdots \mid\left(-\lambda_{p} D+A\right)^{n-1} B\right]=n \quad \forall p \geq 1,
$$

where the $\lambda_{p}$ are the eigenvalues of the Dirichlet Laplacian $-\Delta$; see [2] for the proof.

Furthermore, by combining the results in $[2,17]$, it is possible to deduce a Carleman-like inequality for the solutions to the adjoint of (5.31). Then, the arguments in Section 2 can be adapted to give a hierarchic control result similar to Theorem 1.1. The details will be given in a forthcoming paper.

Acknowledgements. The last version of this paper was written as the third author was at a sabbatical leave as Visiting Professor at the Universidade Federal de Paraíba, Brazil.

\section{REFERENCES}

[1] V. Alexéev, S. Fomine and V. Tikhomirov, Commande optimale, Mir, Moscow (1982).

[2] F. Ammar-Kodjha, A. Benabdallah, M. González-Burgos and L. de Teresa, Recent results on the controllability of linear coupled parabolic problems: a survey. Math. Control Relat. Fields 1 (2011) 267-306.

[3] F. Araruna, B.S.V. Araújo and E. Fernández-Cara, Stackelberg-Nash null controllability for some linear and semilinear degenerate parabolic equations. Math. Control Signals Syst. 30 (2018) Art. 14, 31 pp.

[4] F. Araruna, E. Fernández-Cara, S. Guerrero and M.C. Santos, New results on the Stackelberg-Nash exact control of linear parabolic equations. Systems Control Lett. 104 (2017) 78-85.

[5] F. Araruna, E. Fernández-Cara and M.C. Santos, Stackelberg-Nash exact controllability for linear and semilinear parabolic equations. ESAIM: COCV 21 (2015) 835-856.

[6] F. Boyer, On the penalised HUM approach and its applications to the numerical approximation of null-controls for parabolic problems, in CANUM 2012, Super-Besse, ESAIM Proc., EDP Sci., Les Ulis (2013).

[7] J.C. Cox and M. Rubinstein, Options Markets. Prentice-Hall, Englewood Cliffs, NJ (1985).

[8] J.I. Díaz, On the von Neumann problem and the approximate controllability of Stackelberg-Nash strategies for some environmental problems. Rev. R. Acad. Cien., Serie A. Math. 96 (2002) 343-356.

[9] J.I. Díaz and J.-L. Lions, On the approximate controllability of Stackelberg-Nash strategies. Ocean circulation and pollution control: a mathematical and numerical investigation (Madrid, 1997). Springer, Berlin (2004) 17-27.

[10] E. Fernández-Cara and S. Guerrero, Global Carleman inequalities for parabolic systems and applications to controllability. SIAM J. Control Optim. 45 (2006) 1395-1446.

[11] A.V. Fursikov and O.Y. Imanuvilov, Controllability of evolution equations, Lecture Note Series 34. Research Institute of Mathematics, Seoul National University, Seoul (1996).

[12] I.V. Girsanov, Lectures on mathematical theory of extremum problem, Lecture notes in Economics and mathematical systems 67. Springer-Verlag, Berlin (1972).

[13] F. Guillén-González, F.P. Marques-Lopes and M.A. Rojas-Medar, On the approximate controllability of Stackelberg-Nash strategies for Stokes equations. Proc. Amer. Math. Soc. 141 (2013) 1759-1773.

[14] O.Yu. Imanuvilov, J.-P. Puel and M. Yamamoto, Carleman estimates for parabolic equations with nonhomogeneous boundary conditions. Chin. Ann. Math. Ser. B 30 (2009) 333-378.

[15] J.-L. Lions, Contrôle de Pareto de systèmes distribués. Le cas d'évolution. C.R. Acad. Sc. Paris, série I 302 (1986) $413-417$.

[16] J.-L. Lions, Some remarks on Stackelberg's optimization. Math. Models Methods Appl. Sci. 4 (1994) $477-487$.

[17] Y. Liu, T. Takahashi and M. Tucsnak, Single input controllability of a simplified fluid-structure interaction model. ESAIM: COCV 19 (2013) 20-42.

[18] V. Pareto, Cours d'économie politique. Rouge, Laussane, Switzerland (1896).

[19] A.M. Ramos, R. Glowinski and J. Periaux, Pointwise control of the Burgers equation and related Nash equilibria problems: A computational approach. J. Optim. Theory Appl. 112 (2001) 499-516.

[20] A.M. Ramos, R. Glowinski and J. Periaux, Nash equilibria for the multiobjective control of linear partial differential equations. J. Optim. Theory Appl. 112 (2002) 457-498.

[21] S.M. Ross, An introduction to mathematical finance. Options and other topics. Cambridge University Press, Cambridge (1999). 
[22] P. Wilmott, S. Howison, J. Dewynne, The mathematics of financial derivatives. Cambridge University Press, New York (1995).

\section{Subscribe to Open (S2O) A fair and sustainable open access model}

This journal is currently published in open access under a Subscribe-to-Open model (S2O). S2O is a transformative model that aims to move subscription journals to open access. Open access is the free, immediate, online availability of research articles combined with the rights to use these articles fully in the digital environment. We are thankful to our subscribers and sponsors for making it possible to publish this journal in open access, free of charge for authors.

Please help to maintain this journal in open access!

Check that your library subscribes to the journal, or make a personal donation to the S2O programme, by contacting subscribers@edpsciences.org

More information, including a list of sponsors and a financial transparency report, available at: https://www.edpsciences.org/en/maths-s2o-programme 